\title{
Functional neuroimaging studies of category specificity in object recognition: A critical review and meta-analysis
}

\author{
JANE E. JOSEPH \\ University of Kentucky College of Medicine, Lexington, Kentucky
}

\begin{abstract}
Functional neuroimaging studies in which the cortical organization for semantic knowledge has been addressed have revealed interesting dissociations in the recognition of different object categories, such as faces, natural objects, and manufactured objects. The present paper critically reviews these studies and performs a meta-analysis of stereotactic coordinates to determine whether category membership predicts patterns of brain activation across different studies. This meta-analysis revealed that, in the ventral temporal cortex, recognition of manufactured objects activates more medial aspects of the fusiform gyrus, as compared with natural object or face recognition. Face recognition activates more inferior aspects of the ventral temporal cortex, as compared with manufactured object recognition. The recognition task used - viewing, matching, or naming - also predicted brain activation patterns. Specifically, matching tasks recruit more inferior occipital regions than do either naming or viewing tasks, whereas naming tasks recruit more anterior ventral temporal sites than do either viewing or matching tasks. These findings indicate that the cognitive demands of a particular recognition task are as predictive of cortical activation patterns as is category membership.
\end{abstract}

Visual recognition is the capacity to define, describe, name, or otherwise identify an object or stimulus through vision. Visual recognition is used here as a very general term to refer collectively to a range of cognitive tasks that involve processing perceptual and semantic information associated with objects presented visually. Although the term is used very generally in the present context, other authors have made important distinctions between what is meant by recognition and other types of cognitive tasks, such as naming (Tranel \& A. R. Damasio, 1999; Tranel, H. Damasio, \& A. R. Damasio, 1997). These distinctions are motivated by the cognitive-processing differences among various types of tasks and by neuroanatomical evidence from human lesion studies that the processes are dissociable. In the present context, the term visual recognition is used when referring to the collective tasks of defining, describing, naming, matching, and viewing objects. In discussions of particular studies, terms referring to the specific type of task or tasks are used.

The neural substrates that support visual recognition are undoubtedly as complex as the process itself. Clues to the neural substrates for visual recognition were initially provided by studies that characterized cognitive deficits following cortical insult. In general, damage to inferior and posterior temporal regions or sites at the junction of the

I thank Charles D. Smith and C. Mark Wessinger for their helpful comments on an earlier draft of this manuscript. Correspondence concerning this article should be addressed to J. E. Joseph, Department of Anatomy and Neurobiology, University of Kentucky College of Medicine, 800 Rose Street, Davis-Mills Building Room 308, Lexington, KY 40536 (e-mail: jjoseph@ pop.uky.edu). temporal, occipital, and parietal lobes can lead to severe visual recognition difficulties (A. R. Damasio, Tranel, \& H. Damasio, 1990; McCarthy \& Warrington, 1990; Tranel, H. Damasio, \& A. R. Damasio, 1997). Visual agnosia refers to a deficit in recognizing objects through vision that typically cannot be attributed to impaired sensory processing or general intellectual decline (Bauer, 1993). Often, objects that cannot be recognized through vision can be recognized through other sensory modalities, such as touch. In category-specific visual agnosia, not all categories of objects are equally compromised. Prosopagnosia is a specific type of visual recognition deficit restricted to faces, but not to other object categories. In some cases, the recognition impairment is so profound that individuals cannot recognize their own faces in the mirror (Pallis, 1955). In addition to face recognition being dissociated from object recognition, recognition of natural and manufactured objects appear to be dissociated. For example, some patients have difficulty describing, defining, and naming natural objects, such as animals and plants, but no difficulties with these same tasks applied to manufactured objects, such as tools and utensils (Warrington \& Shallice, 1984). The opposite dissociation-impaired recognition of manufactured objects but intact recognition of natural objects - has also been reported (Warrington \& McCarthy, 1994). This apparent double dissociation in the recognition of natural and manufactured object categories has been a popular focus in the study of visual agnosia, but the numerous case and group lesion studies on this topic are beyond the scope of this review (for indepth discussions of this topic, see Caramazza \& Shelton, 1998; Farah, 1990; Forde \& Humphreys, 1999). The fact 
that cortical insult can lead to such dissociations is not only intriguing, it may also elucidate the functional and anatomical organization of semantic knowledge.

Although the clinical phenomenon of category-specific deficits is well established, there is continuing debate as to the cognitive nature of these deficits. This debate is largely focused on the factor or factors that may be driving the category distinctions that lead to dissociations. In the sensory-functional account (Warrington \& Shallice, 1984), natural objects are largely organized by their visual features, whereas manufactured objects are organized by their functions. For example, in this approach, birds tend to cluster together into a category because they are similarly shaped, but objects like forks and spoons are classed together owing to their common function in eating. The organizing principle for semantic knowledge, then, is the type of information needed to recognize different object classes, rather than category membership itself. If the cortical systems used to represent visual (or other sensory) knowledge is damaged, natural object recognition suffers. If there is damage to areas of the cortex that are used to represent functional attributes, manufactured object recognition suffers.

One problem with the sensory-functional approach is that natural and manufactured object categories differ from each other along several dimensions, including familiarity, word frequency, visual complexity, and structural similarity (Humphreys \& Riddoch, 1987; Tranel, Logan, Frank, \& A. R. Damasio, 1997). Therefore, it is difficult to attribute differences in recognizing different categories to any single factor unless all of these other variables are controlled. Another problem is that there are notable exceptions to the strict living/nonliving distinction. For example, naming of animals can be damaged separately from naming of fruits and vegetables (Caramazza \& Shelton, 1998), even though both are natural categories. Some investigators argue that category-specific effects can be completely explained by some extraneous factor and, consequently, that category-specific deficits are artifactual (Gaffan \& Heywood, 1993). Still others argue that variables other than category membership may underlie category-specific impairments but these variables are not extraneous; in fact, they are central to the process of object or face recognition. For example, natural objects may overlap in terms of their shape or structure more so than manufactured objects (Bruce \& Humphreys, 1994; A. R. Damasio, H. Damasio, \& Van Hoesen, 1982; Humphreys, Riddoch, \& Quinlan, 1988). A third stance argues that even when these other factors are controlled, category-specific impairments persist (Kurbat \& Farah, 1998). Although the debate is not resolved, this body of literature has been invaluable in outlining some of the critical factors that may underlie category-specific recognition deficits and in providing theoretical foundations for understanding the organization of semantic knowledge.

In addition to the lack of consensus concerning the cognitive nature of category-specific impairments, there is not complete agreement as to the neuroanatomical loci of these deficits. The lesions or areas of atrophy are so large that they may encompass entire lobes. However, H. Damasio, Grabowski, Tranel, Hichwa, and Damasio (1996) conducted a group study of 127 patients with focal lesions and deficits in naming animals, tools, or faces to elucidate the neural sites for category-specific naming impairments. In that study, a relationshipbetween locus of lesion and type of category-specific impairment was demonstrated: Patients with temporal pole lesions had impaired face naming, patients with anterior inferior temporal lesions had impaired animal naming, and patients with occipito-temporal lesions had impaired tool naming. By using PET in a parallel study, brain activation patterns were measured in healthy adult volunteers while they named faces, animals, or tools. Face naming activated a temporal pole region, animal naming activated an anterior inferior temporal region, and tool naming activated both anterior and posterior inferior temporal regions. All the object categories activated a temporal pole region that was different from the temporal pole region activated by faces only. This combined human lesion and functional imaging study suggests that different cortical regions are preferentially involved in the naming of different object categories. The advantage of using functional neuroimaging techniques, such as PET and $\mathrm{FMRI}$, is that they may provide greater spatial precision than lesion studies for identifying the neural substrates of categoryspecific recognition.

The purpose of this literature review is to examine the functional neuroimaging studies to date that have addressed category-specific processing in object recognition, where recognition refers to three general types of tasks: viewing, matching, and naming of objects. Three major classes of objects will be examined-faces, natural objects, and manufactured objects-because the functional neuroimaging and human lesion studies conducted on this topic have largely focused on these categories. The review will critically address the following questions. (1) Is there a consensus as to the neural substrates of category-specific processing across functional neuroimaging studies? (2) Have the analyses used in these studies sufficiently tested category-specific hypotheses? (3) Have the important cognitive factors outlined in cognitive neuropsychological research been sufficiently manipulated in these studies? In addition to evaluating the extant literature on category specificity, the present paper conducts a meta-analysis of stereotactic coordinates reported in those studies, to determine the extent to which category membership predicts the spatial distribution of brain activation. The meta-analysis will also examine the contribution of different types of recognition tasks to the spatial distribution of brain activation patterns associated with visual recognition. This analysis will provide insights as to the neural substrates associated with categoryspecific recognition impairments and visual recognition.

\section{Overview of the Studies}

In the past 8 years, over two dozen neuroimaging studies have addressed the question of category specificity, 
using a variety of imaging techniques and tasks (Allison et al., 1994; Cappa, Perani, Schnur, Tettamanti, \& Fazio, 1998; Chao, Haxby, \& Martin, 1999; Chao \& Martin, 2000; H. Damasio et al., 1996; Gauthier, Skudlarski, Gore, \& Andersen, 2000; Gauthier, Tarr, Anderson, Skudlarski, \& Gore, 1999; Gauthier, Tarr, et al., 2000; Gerlach, Law, Gade, \& Paulson, 1999; Grabowski, H. Damasio, \& A. R. Damasio, 1998; Haxby et al., 1999; Ishai, Ungerleider, Martin, \& Haxby, 2000; Kanwisher, McDermott, \& Chun, 1997; Krieman, Koch, \& Fried, 2000; Martin, Wiggs, Ungerleider, \& Haxby, 1996; Moore \& Price, 1999; Mummery, Patterson, Hodges, \& Price, 1998; Nakamura et al., 2000; Perani et al., 1999; Puce, Allison, Asgari, Gore, \& McCarthy, 1996; Rossion et al., 2000; Smith et al., 2001; Spitzer et al., 1998; Spitzer, Kwong, Kennedy, Rosen, \& Belliveau, 1995; Tempini et al., 1998; Thompson-Schill, Aguirre, D'Esposito, \& Farah, 1999). In the majority of these studies, neurologically unimpaired individuals perform recognition tasks on different categories of objects. The numerous studies on this topic are considered throughout this paper, but not all of the studies were selected for inclusion in the meta-analyses.

One criterion for inclusion was an emphasis on recognition of natural objects, manufactured objects, and faces, because the controversies in the literature typically center on the face/object dichotomy or on the natural/ manufactured dichotomy (also referred to as the living/ nonliving distinction). Consequently, studies with a major focus on the visual categories of colors, letters, and digits were not included. A second criterion for inclusion was that the study directly compared different categories of objects within the same experiment. For example, a study that directly compared faces with objects or natural with manufactured objects was included. Studies that only compared a target category (natural objects, manufactured objects, or faces) with a baseline resting condition or a control condition that did not use stimuli from meaningful categories were not included in the analyses. Some studies contrasted one of the target categories both with a baseline task and with another target category, but results from only the most selective contrasts are included in the present analysis. The reason for this stipulation was that directly comparing two categories is a more selective contrast. In addition, control tasks vary widely across studies, which can raise some problems in interpreting contrasts that do not have similar baseline conditions. A previous review paper on functional neuroimaging studies of visual recognition did not observe strong patterns of brain activation as a function of object category, task, or stimulus (Farah \& Aguirre, 1999), but these brain activation patterns were not assessed statistically. The authors argued that the lack of convergence across studies was due to the wide range of control tasks used. They suggested that a more fruitful approach to understanding the neural correlates of visual recognition is to directly contrast different object categories. This suggestion is adopted for the present analysis. Although not included in the meta-analysis, studies that did not make direct comparisons among faces, natural objects, or manufactured objects will be discussed in the text.

A third criterion for inclusion was that the task used in the study captured some aspect of object recognition and identification, rather than focused on other types of cognitive processing, such as memory or linguistic processing. To simplify the meta-analysis, the tasks used in the various studies are broadly divided into three groups: viewing, matching, or naming tasks. A fourth criterion for inclusion in the present review was that the results were reported using the standardized stereotactic system of Talairach and Tournoux (1988). These Talairach coordinates characterize a locus of brain activation along three spatial axes: left-right (or medial-lateral; $x$-coordinates), anterior-posterior ( $y$-coordinates), and inferior-superior (z-coordinates). This criterion necessarily excludes any studies that use electrical techniques and includes studies using hemodynamic techniques, such as PET or fMRI. The reason for this stipulation was that the meta-analyses are conducted on Talairach coordinates to determine the spatial distribution of category-specific responses.

Fourteen studies met all four criteria above and are listed in Table 1. These studies manipulated and directly contrasted at least two of the three major categories of objects considered here. Natural object categories included animals, fruits, vegetables, and body parts; manufactured object categories included tools and utensils, chairs, houses, vehicles, appliances, musical instruments, and scenes that included buildings; faces included unfamiliar or familiar faces. The tasks used in these studies included viewing (passively viewing pictures of objects or faces), matching (matching two pictures or matching a picture to a remembered picture), naming (overt or covert naming of pictures), and semantic retrieval or association tasks. The semantic retrieval tasks were grouped with the naming tasks for the meta-analysis because both require retrieving semantic information from memory.

Tables 2-5 list the stereotactic coordinates reported in occipital, ventral temporal, temporal, and frontal regions, respectively, from the 14 core studies. Although the key studies reported activation loci other than those listed in Tables 2-5, these loci were often not reported in more than 2 studies, so they are not included in the present analysis. For a given contrast in a particular study, several Talairach coordinates could be reported, but only one coordinate is included in Tables 2-5 (typically, the one with the higher statistical $z$-score value). Some loci within a study are nearly identical and seem redundant, but these loci were taken from different contrasts, tasks, or experiments within the same study (see Table 1 for more details on the different contrasts used in various studies). Figures 1 and 2 graphically depict the major activation loci for the occipital/ventral temporal regions and the temporal/frontal regions, respectively (in some cases, the coordinates reported in a single study for the same object category were so close together that not all of the coordinates were included in the figures). The occipital regions (Table 2, Figure 1) include medial occip- 
Table 1

The 14 Studies Included in the Meta-Analysis

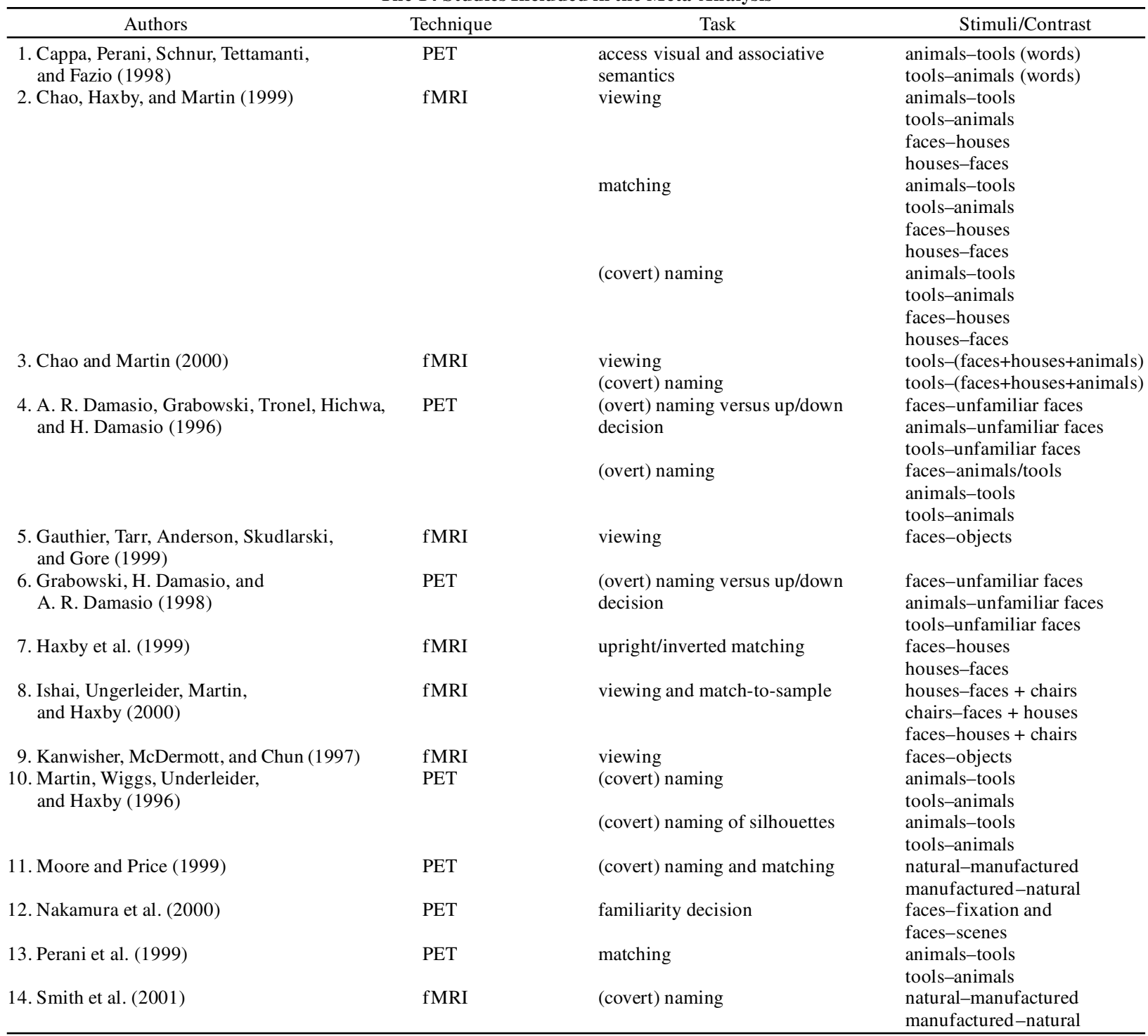

ital structures (lingual gyrus, calcarine sulcus, and the cuneus) as well as the inferior occipital/posterior fusiform cortex. The ventral temporal regions (Table 3, Figure 1) include the mid-fusiform gyrus, inferior temporal cortex, anterior fusiform gyrus, and the temporal pole. Other temporal regions (Table 4, Figure 2) are the middle and superior temporal cortex. Frontal regions (Table 5, Figure 2) include the inferior frontal, middle frontal, and precentral gyri. A cursory inspection of Figures 1 and 2 reveals some interesting tendencies for dissociated cortical involvement in the recognition of different object categories. For example, inspection of the activation loci in occipital regions reveals that natural and manufactured object recognition recruit both the medial occipital cortex and posterior fusiform regions but that face-related activation is restricted to posterior fusiform regions. Whether this and other patterns are robust and consistent across studies is addressed in the next section.

\section{Meta-Analysis of Stereotactic Coordinates}

To determine whether these patterns are meaningful and statistically significant, a meta-analysis of the reported Talairach coordinates in Tables 2-5 was conducted. In addition to category membership, the type of recognition task was entered into the model. A third variable entered into the model was brain region. The reason for including brain region as a variable was to determine whether different brain areas show different spatial distributions of brain activation as a function of category or task. The $143 \mathrm{co}-$ ordinates in Tables 2-5 were submitted to a $3 \times 3 \times 4$ multivariate analysis of variance (MANOVA) with $x, y$, and $z$ stereotactic coordinates as the dependent variables 
Table 2

Talairach Coordinates Reported in Occipital Regions

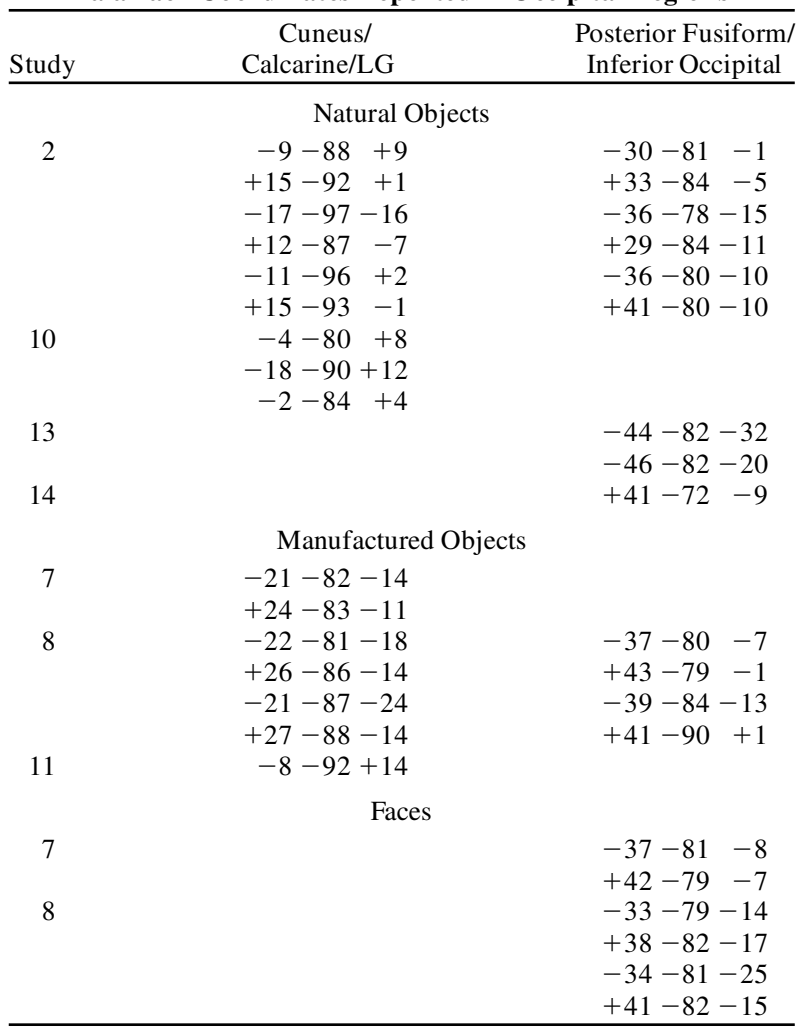

Note-Study numbers refer to the list of studies in Table 1. LG, lingual gyrus.

and category (faces, natural objects, or manufactured objects), task (viewing, matching, or naming), and region (occipital, ventral temporal, temporal, and frontal) as the independent variables. ${ }^{1}$ The absolute value of the $x$-coordinate was used, rather than the signed value, because initial inspection of the average signed $x$ value revealed a mean close to zero, indicating that across all studies, activation was distributed bilaterally. Results from both the univariate and the multivariate tests (using Wilks's lambda for the $F$ values) are reported. The alpha level used to assess statistical significance was .05 for all main effects and interactions, and post hoc comparisons using Tamahane's F2 test ${ }^{2}$ were conducted on means for which univariate main effects emerged as significant.

The overall model was significant $[F(3,112)=429.1]$, as were the main effects of category $[F(6,224)=3.0]$, task $[F(6,224)=7.7]$, and brain region $[F(9,272)=$ 55.3]. Two interactions were significant: category $\times$ area $[F(18,317)=1.7]$ and task $\times$ area $[F(18,317)=1.7]$. The overall mean locus of activation across all categories, tasks, and regions was $x= \pm 39, y=-44, z=-1$, which falls squarely in the mid-fusiform gyrus. This is not surprising given that almost half of the coordinates submitted to the MANOVA came from the ventral temporal cortex, whereas the remaining coordinates were more evenly distributed among the other three brain regions. This will naturally bias the mean $x, y$, and $z$ val- ues toward a locus in the ventral temporal cortex, and the main effects will be biased toward patterns in ventral temporal regions; therefore, interpreting the main effects will raise problems. The reason for such an unbalanced design is that, in the 14 core studies, not all brain regions

Table 3

Talairach Coordinates Reported in Ventral Temporal Regions

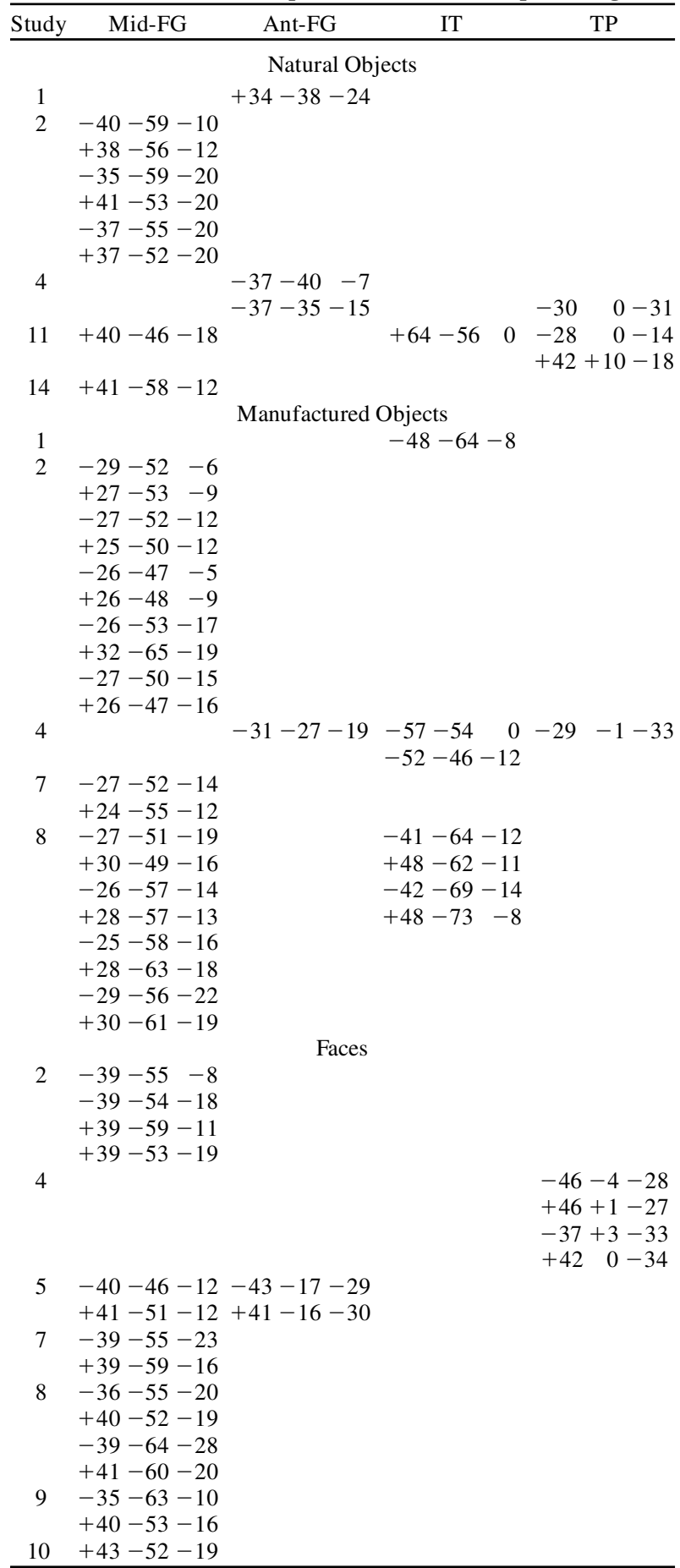

Note-Study numbers refer to the list of studies in Table 1. Mid-FG, middle fusiform gyrus; Ant-FG, anterior fusiform gyrus; IT, inferior temporal cortex; TP, temporal pole. 
Table 4

Talairach Coordinates Reported in Middle and Superior Temporal Regions

\begin{tabular}{|c|c|c|}
\hline Study & STS/STG & MTG \\
\hline \multicolumn{3}{|c|}{ Natural Objects } \\
\hline 2 & $\begin{array}{l}-42-59+19 \\
+53-54+16 \\
+43-61+12 \\
-43-63+8 \\
+52-59+15 \\
+70-42+8\end{array}$ & \\
\hline 11 & Manufactured Objects & \\
\hline 1 & $+52-16+16$ & \\
\hline 2 & & $\begin{array}{r}-46-55+3 \\
+40-53 \quad 0 \\
-47-54+6 \\
+46-56+4 \\
-45-57+7 \\
+50-54+6 \\
-36-50+4 \\
-40-62+4\end{array}$ \\
\hline 10 & Faces & \\
\hline 2 & $\begin{array}{l}-42-59+19 \\
+53-54+16 \\
-48-57+9 \\
+51-55+12\end{array}$ & \\
\hline 4 & & $-56-14+9$ \\
\hline 7 & $\begin{array}{l}-52-61+4 \\
+43-57+12\end{array}$ & \\
\hline 8 & $\begin{array}{l}-53-45-4 \\
+54-47+9 \\
-53-53+1 \\
+51-59+18\end{array}$ & \\
\hline
\end{tabular}

Note-Study numbers refer to the list of studies in Table 1. STS, superior temporal sulcus; STG, superior temporal gyrus; MTG, middle temporal gyrus.

Table 5

Talairach Coordinates Reported in Frontal Regions

\begin{tabular}{|c|c|c|c|}
\hline Study & IFG & $\begin{array}{l}\text { IFG/PCG } \\
\text { (Premotor) }\end{array}$ & MFG \\
\hline \multicolumn{4}{|c|}{ Natural Objects } \\
\hline $\begin{array}{r}1 \\
6 \\
10 \\
14\end{array}$ & $\begin{array}{l}-44+22+3 \\
-26+28+16 \\
-41+12-11 \\
+37+17-10\end{array}$ & $-26-6+24$ & $+30+50+12$ \\
\hline \multicolumn{4}{|c|}{ Manufactured Objects } \\
\hline 3 & $-30+17+9$ & $\begin{array}{l}-42+6+23 \\
-50+3+25\end{array}$ & \\
\hline 6 & $-44+23+8$ & & $-28+53+16$ \\
\hline 10 & $-52+10+20$ & $\begin{array}{ll}-48 & 0+20 \\
-42 & 0+20\end{array}$ & \\
\hline 13 & $-54+8+8$ & & \\
\hline \multicolumn{4}{|c|}{ Faces } \\
\hline 6 & $-44+22$ & & $-34+47+14$ \\
\hline
\end{tabular}

Note-Study numbers refer to the list of studies in Table 1. IFG, inferior frontal gyrus; PCG, precentral gyrus; MFG, middle frontal gyrus.

were sampled with equal frequency. Most of the fMRI studies did not provide whole-brain coverage but, instead, imaged only posterior regions (Chao et al., 1999; Haxby et al., 1999; Ishai et al., 2000) or only the occipital cortex and the temporal cortex (Gauthier et al., 1999; Kan- wisher et al., 1997). In addition, analyses are often further restricted to particular regions of interest (ROIs) or the contrasts of interest are first masked by contrasts establishing an experimental effect (Chao et al., 1999; Chao \& Martin, 2000; H. Damasio et al., 1996; Gauthier et al., 1999; Haxby et al., 1999; Ishai et al., 2000; Kanwisher et al., 1997; Nakamura et al., 2000; Perani et al., 1999), which can further bias the results toward a particular average brain region in the meta-analysis (this point is discussed in more detail later).

Although the unbalanced design biased the results toward a common locus in the ventral temporal cortex, both task and category interacted with brain region, indicating that different patterns emerge in different brain regions as a function of category or task. Table 6 and Figure 3 show the average activation loci for the three categories by brain region, whereas Table 7 and Figure 4 show the average loci of activation for the three tasks by brain region. Given the interactions with brain region, a MANOVA was repeated for the four brain regions separately. Of course, in these $3 \times 3$ MANOVAs, only category and task were manipulated. In occipital regions, the overall model was significant $[F(3,27)=1,715.9]$, as was the task effect $[F(6,54)=3.5]$, but not the category effect or the interaction. Univariate tests revealed that the inferior-superior dimension was affected by task $[F(2,29)=2.8]$. As is

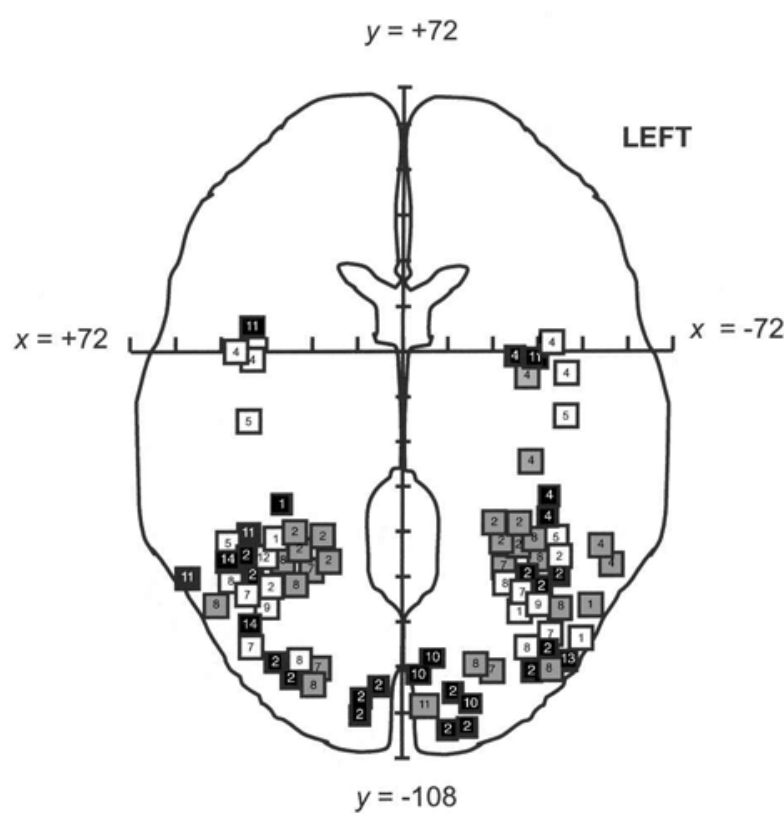

Natural objects

Manufactured objects

Faces

Figure 1. Activation loci in occipital and ventral temporal regions associated with natural object (black squares), manufactured object (gray squares), and face (white squares) recognition tasks overlaid on an axial slice representation collapsed in the inferior-superior dimension. The number within each square refers to the study, as listed in Table 1. 

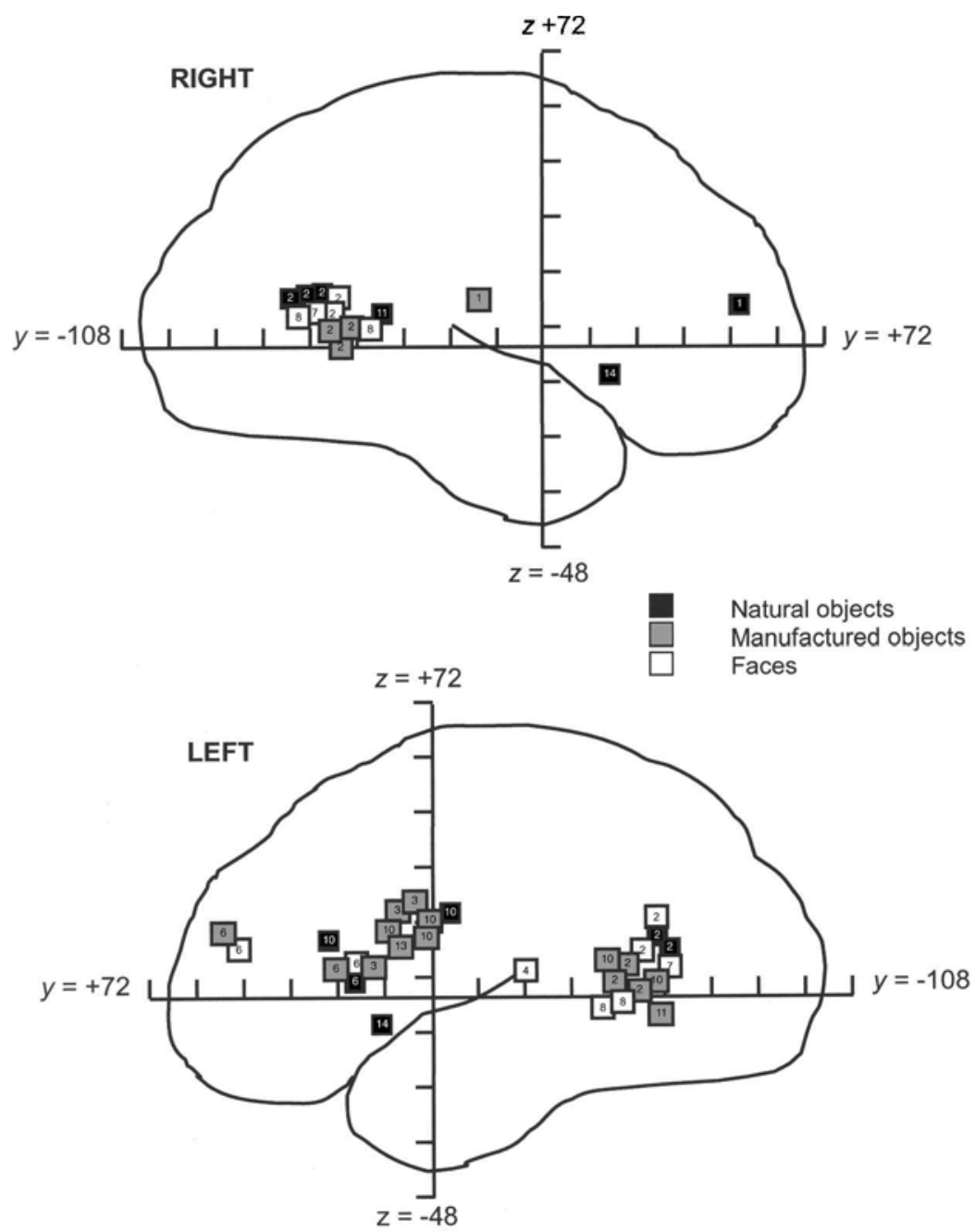

Figure 2. Activation loci in temporal and frontal regions associated with natural object (black squares), manufactured object (gray squares), and face (white squares) recognition tasks overlaid on sagittal slice representations. The number within each square refers to the study, as listed in Table 1.

shown in Figure 4, matching tasks activate more inferior occipital sites (mean $z$-coordinate $=-14$ ) than do either viewing (mean $z$-coordinate $=+1$ ) or naming (mean $z$ coordinate $=+6$ ) tasks, but naming and viewing tasks do not activate significantly different loci in the inferiorsuperior dimension.

In ventral temporal regions, the overall model was significant $[F(3,54)=1,093.3]$, as were the main effects of category $[F(6,108)=8.3]$ and task $[F(6,108)=10.3]$, but not the interaction. Univariate tests revealed significant category effects along the medial-lateral axis $[F(2,56)=7.8]$, and the inferior-superior axis $[F(2,56)=$ $8.5]$ and a marginal effect along the anterior-posterior axis $[F(2,56)=3.0, p=.057$; Figure 3$]$. Post hoc tests revealed that average manufactured object activation (mean $x$-coordinate $= \pm 32$ ) was situated more medially than average face (mean $x$-coordinate $= \pm 41$ ) or natural object (mean $x$-coordinate $= \pm 39$ ) activation but that face and natural object activations were not different in the medial-lateral dimension. Post hoc tests also revealed that faces activated more inferior aspects of ventral temporal regions (mean $z$-coordinate $=-21$ ) than did manufactured objects (mean $z$-coordinate $=-13$ ) but that faces and natural objects do not activate different aspects along the inferior-superior axis. No significant differences emerged for the anterior-posterior axis according to post hoc tests. For the significant task effect in ventral temporal regions (Figure 4), univariate tests showed that activation in both the anterior-posterior $[F(2,56)=$ $14.9]$ and the inferior- superior $[F(2,56)=6.7]$ dimensions were predicted by task. However, the post hoc tests revealed differences only in the anterior-posterior dimension. Naming tasks (mean $y$-coordinate $=-28$ ) activated more anterior ventral temporal regions than did either viewing (mean $y$-coordinate $=-51$ ) or matching (mean $y$-coordinate $=-57)$ tasks, but viewing and matching 
Table 6

Mean $x, y$, and $z$ Values by Area and Category (With Standard Errors)

\begin{tabular}{|c|c|c|c|c|c|c|c|c|}
\hline \multirow[b]{3}{*}{ Area } & \multirow[b]{3}{*}{ Category } & \multirow[b]{3}{*}{$n$} & \multicolumn{6}{|c|}{ Talairach Coordinate } \\
\hline & & & \multicolumn{2}{|c|}{$x$} & \multicolumn{2}{|c|}{$y$} & \multicolumn{2}{|c|}{$z$} \\
\hline & & & $M$ & $S E$ & $M$ & $S E$ & $M$ & $S E$ \\
\hline \multirow[t]{3}{*}{ Occipital } & faces & 6 & \pm 38 & 5 & -81 & 2 & -14 & 3 \\
\hline & manufactured & 11 & \pm 19 & 6 & -88 & 3 & +1 & 4 \\
\hline & natural & 18 & \pm 24 & 3 & -85 & 1 & -6 & 2 \\
\hline \multirow[t]{3}{*}{ Ventral temporal } & faces & 21 & \pm 41 & 2 & -37 & 4 & -21 & 1 \\
\hline & manufactured & 29 & \pm 32 & 2 & -49 & 4 & -13 & 1 \\
\hline & natural & 15 & \pm 39 & 3 & -50 & 6 & -15 & 2 \\
\hline \multirow[t]{3}{*}{ Other temporal } & faces & 11 & \pm 51 & 3 & -42 & 4 & +11 & 2 \\
\hline & manufactured & 9 & \pm 45 & 2 & -52 & 4 & +5 & 2 \\
\hline & natural & 6 & \pm 49 & 3 & -57 & 5 & +13 & 2 \\
\hline \multirow[t]{3}{*}{ Frontal } & faces & 2 & \pm 39 & 6 & +35 & 13 & +10 & 7 \\
\hline & manufactured & 9 & \pm 46 & 4 & +9 & 9 & +16 & 5 \\
\hline & natural & 6 & \pm 34 & 3 & +20 & 8 & +6 & 4 \\
\hline
\end{tabular}

Note- $n$, number of observations; $x$, medial-lateral coordinate; $y$, anterior-posterior coordinate; $z$, inferior-superior coordinate.

did not activate significantly different loci in the anteriorposterior dimension.

In other temporal regions, the overall model was significant $[F(3,15)=981.6]$, as was the effect of category $[F(6,30)=3.0]$. Univariate tests revealed that only the inferior-superior axis showed category effects $[F(2,17)=$ 4.6; Figure 3]. Specifically, the locus for manufactured object recognition tended toward the middle temporal cortex (mean $z$-coordinate $=+5$ ), but the locus for natural object recognition tended toward the superior temporal cortex (mean $z$-coordinate $=+13$ ). Manufactured object and face recognition loci were not different (mean $z$-coordinate $=+11$, for face recognition). In frontal regions, the overall model was significant $[F(3,12)=19.7]$, but no main effects or interactions were significant. The number of observations in frontal regions is quite low (Tables 6 and 7), and some cells of the design have only one case, which can lower the power to detect effects of interactions. Null effects of category or task in frontal regions, then, may simply be attributed to lower power.

This meta-analysis of stereotactic coordinates conducted by brain region revealed five main findings. (1) Brain activation patterns in the ventral temporal cortex are distributed differently for the three categories of objects examined here-faces, natural objects, and manufactured objects. Manufactured object recognition tends to recruit more medial ventral temporal structures than does either natural object or face recognition. (2) Face recognition dissociates from manufactured object recognition by activating more inferior aspects of the ventral temporal cortex. (3) In other temporal regions, manufactured and natural object recognition dissociate from each other by activating middle and superior temporal regions, respectively. (4) The type of recognition task used also revealed dissociated patterns of brain activation. Matching tasks activate more inferior occipital regions than do either viewing or naming tasks. (5) In the ventral temporal cortex, naming tasks activate more anterior regions than do view- ing or matching tasks. With regards to category effects, manufactured object recognition tends to dissociate from other object categories, but none of the contrasts demonstrated dissociations of natural object and face recognition. The finding that task effects were as strong as category effects is important and will be discussed in more detail later.

Although the meta-analysis results are informative, they should be considered only a first-pass approximation of brain activation patterns related to category and task specificity, owing to the unequal cell sizes and low power for detecting some effects. To supplement this metaanalysis, the studies addressing category specificity are now addressed in more detail, focusing on the three main questions presented in the introduction. For each major object category (addressed in a separate section), results reported in the literature will be assessed as to (1) the consistency of the findings across studies for different object categories, (2) the adequacy of the analyses used for hypothesis testing, and (3) the isolation of the critical cognitive factors that may drive category differences.

\section{Areas Specific to Face Recognition}

To determine cortical specialization for face recognition, studies have examined $\mathrm{fMRI}$ and PET responses to pictures of unfamiliar faces, as compared with other object categories (Chao et al., 1999; Gauthier et al., 1999; Haxby et al., 1999; Ishai et al., 2000; Kanwisher et al., 1997), compared familiar with unfamiliar face processing (H. Damasio et al., 1996), or compared familiar and unfamiliar face recognition with familiar and unfamiliar scene recognition (Nakamura et al., 2000). Tasks have included passive viewing, matching to sample, familiarity decisions, and covert and overt naming. Figure 1 graphically summarizes the Talairach coordinates associated with face recognition (white boxes) in the occipital and ventral temporal cortices, and Tables 2 and 3 list these coordinates. In these major cortical regions, there is a strong concentration of face-related activation in the midfusiform gyrus, and in nearly all studies, this activation is bilateral. However, faces also recruit anterior fusiform regions, as well as the temporal pole. Figure 2 summarizes the Talairach coordinates associated with face activation in frontal and temporal regions, and Tables 4 and 5 list these coordinates. Faces activate superior temporal structures bilaterally, the left middle temporal cortex, and the left middle and inferior frontal gyrus. The concentration of activation from faces in frontal and temporal structures is not as great as that in ventral temporal and occipital regions.

Consistency of results across studies. Table 2 and Figure 1 show remarkable consistency of Talairach coordinates in mid-fusiform regions in response to face processing. Specifically, the coordinates listed in Table 2 do not deviate much from each other. Table 8 shows the percentage of studies that detected category-related activation in various brain regions, given that the imaging protocol actually imaged those portions of the brain. Of 


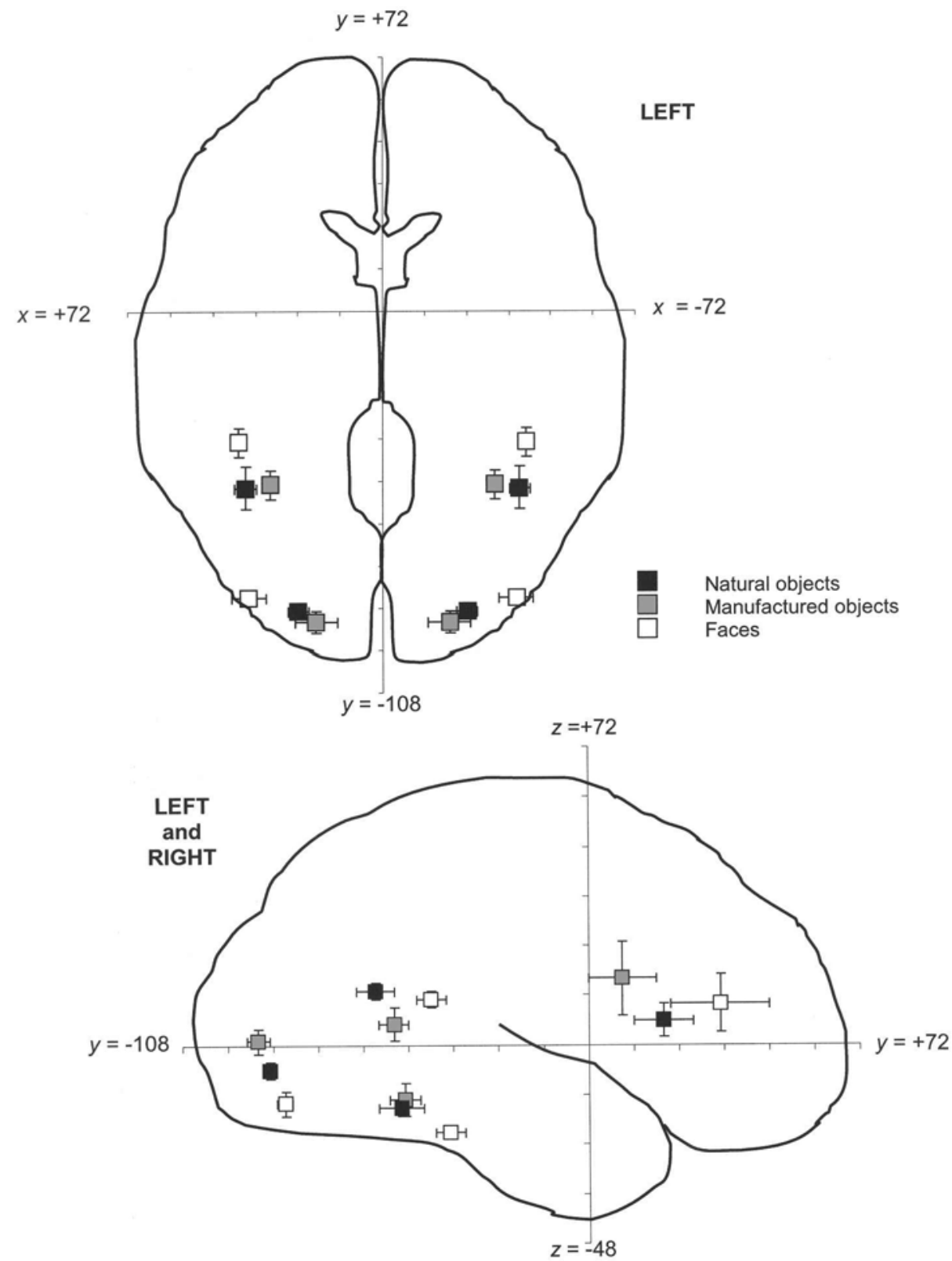

Figure 3. Mean activation loci for natural object (black squares), manufactured object (gray squares), and face (white squares) recognition overlaid on axial and sagittal slice representations. Standard error bars are shown.

the eight studies that isolated face-specific processing in ventral temporal regions, seven of them report activation in the mid-fusiform gyrus. The one study that did not report activation in this region (H. Damasio et al., 1996) was the only one to employ a naming task. The other studies used either viewing or matching tasks. Activation associated with face recognition in the mid-fusiform gyrus is the most consistent finding of all the category effects, according to the present analysis. In addition, several other PET or fMRI studies have isolated this same region, using contrasts of face viewing versus scrambled face viewing (Puce, Allison, Gore, \& McCarthy, 1995), face viewing versus letter string viewing (Puce et al., 1996), face matching versus location matching (Haxby et al., 1994), face identification versus gender identification (Sergent, Ohta, \& MacDonald, 1992), and familiar or unfamiliar face matching versus matching familiar or unfamiliar face names (Tempini et al., 1998). Electrical recording techniques (EEG) have shown that the negative potential occurring in this general region about $200 \mathrm{msec}$ after stimulus onset is more prominent for face viewing, as compared with viewing other objects (Alli- 
Table 7

Mean $x, y$, and $z$ Values by Area and Task (With Standard Errors)

\begin{tabular}{|c|c|c|c|c|c|c|c|c|}
\hline \multirow[b]{3}{*}{ Area } & \multirow[b]{3}{*}{ Task } & \multirow[b]{3}{*}{$n$} & \multicolumn{6}{|c|}{ Talairach Coordinate } \\
\hline & & & \multicolumn{2}{|c|}{$x$} & \multicolumn{2}{|c|}{$y$} & \multicolumn{2}{|c|}{$z$} \\
\hline & & & $M$ & $S E$ & $M$ & $S E$ & $M$ & $S E$ \\
\hline \multirow[t]{3}{*}{ Occipital } & matching & 22 & \pm 33 & 3 & -83 & 1 & -14 & 2 \\
\hline & naming & 9 & \pm 15 & 6 & -88 & 3 & +6 & 4 \\
\hline & viewing & 4 & \pm 22 & 6 & -86 & 3 & 1 & 4 \\
\hline \multirow[t]{3}{*}{ Ventral temporal } & matching & 28 & \pm 36 & 2 & -57 & 4 & -18 & 2 \\
\hline & naming & 23 & \pm 40 & 2 & -28 & 4 & -20 & 1 \\
\hline & viewing & 14 & \pm 35 & 2 & -51 & 5 & -11 & 2 \\
\hline \multirow[t]{3}{*}{ Other temporal } & matching & 11 & \pm 47 & 3 & -57 & 4 & +8 & 2 \\
\hline & naming & 9 & \pm 52 & 3 & -39 & 4 & +9 & 2 \\
\hline & viewing & 6 & \pm 46 & 3 & -56 & 4 & +12 & 2 \\
\hline \multirow[t]{3}{*}{ Frontal } & matching & 1 & \pm 54 & 8 & +8 & 19 & +8 & 10 \\
\hline & naming & 15 & \pm 38 & 3 & +23 & 6 & +11 & 3 \\
\hline & viewing & 1 & \pm 42 & 8 & +6 & 19 & +23 & 10 \\
\hline
\end{tabular}

Note- $n$, number of observations; $x$, medial-lateral coordinate; $y$, anterior-posterior coordinate; $z$, inferior-superior coordinate.

son et al., 1994), or when making upright/inverted decisions with faces, as compared with other objects (Rossion et al., 2000). The fact that this same region is consistently activated across contrasts with a number of different control conditions and techniques is strong evidence for its consistent role in face recognition.

Analyses used to test category-specific hypotheses. The very consistent finding of mid-fusiform activation in response to faces may, on the surface, lead to the conclusion that this region is specific for face recognition. However, in evaluating this claim, one must consider the approaches used for hypothesis testing. The direct contrast of faces versus objects (or other visual categories) should selectively isolate regions that respond more strongly to faces than to objects. Importantly, this contrast alone does not allow the conclusion that a given region is selective for face recognition; it only supports the conclusion that the region responds more strongly to faces than to objects. Other object categories may very well activate this same region to a statistically significant degree, relative to some other control or baseline condition. Kanwisher et al. (1997) have suggested that midfusiform activation is selective for faces, and they called this area the fusiform face area (or FFA), based on their findings that (1) this region is isolated in the direct contrast of faces versus objects and (2) a more medial fusiform region was implicated in the opposite contrast of objects versus faces. They consider these two findings as evidence for a double dissociation and, hence, as evidence for face selectivity in the mid-fusiform cortex. However, this conclusion is premature because no information is provided as to whether objects also activate the FFA more strongly than a baseline task does. If object recognition tasks activate the FFA more strongly than do baseline tasks, the FFA cannot be implicated solely in face processing. Because no information was provided as to whether the object-based activation was greater than that for a baseline task in many of these studies, one cannot appropriately assess the selectivity issue.
Another aspect of hypothesis testing that invites closer examination is the strategy of first localizing an ROI, and then performing subsequent analyses on these ROIs alone, to assess whether they are indeed selective for faces. For example, Kanwisher et al. (1997) isolated the FFA by directly contrasting faces and objects. This region of interest was then used in subsequent analyses to examine the relative contribution of houses, scrambled faces, and human hands to signals in that region. The consistent finding was that the FFA responds more strongly to faces than to the other categories of objects or stimuli tested. However, the initial step of isolating the FFA biases the results toward this conclusion, because the only region being examined in subsequent analyses was one that initially responded more strongly to faces than to other object categories.

Although findings across studies of face recognition have consistently implicated the same mid-fusiform region, this region is not necessarily selective for faces. The analyses to date have only allowed the conclusion that the mid-fusiform "face" area responds more strongly to faces than to other object categories but is not exclusive for faces.

Isolation of critical cognitive factors. Despite the limited conclusions that can be made about face-selective activation in the FFA, Kanwisher et al. (1997) ruled out several alternative explanations for the greater $\mathrm{CMRI}$ response to faces than to objects. First, two-toned face recognition was compared with scrambled two-toned face recognition to rule out the possibility that the FFA responds only to a particular luminance pattern (as would be found with gray-scale faces) or to individual facial features and not to faces as a whole. Second, faces were also compared with human hand recognition to rule out the possibility that the FFA responds to any biological form. Finally, face recognition was compared with house recognition to rule out the possibility that the FFA responds to any set of objects with many different exemplars.

Some investigators, however, have questioned the nature of the response in the FFA. Gauthier et al. (1999) have proposed that the FFA is not selective for the category of faces at all; rather, it is involved in recognition of objects at a more specific or individual level than one would use to recognize other categories. For example, when presented with a picture of an elephant, a likely response to label the picture would be the basic-level (Rosch, Mervis, Gray, Johnson, \& Boyes-Braem, 1976) or entrylevel (Jolicœur, Gluck, \& Kosslyn, 1984) name "elephant." But when presented with a picture of a face, a likely response is to provide the name of the face (e.g., "Bob") rather than to say "face." To test the hypothesis that the FFA is involved when objects are recognized at an individual level of categorization, rather than being specifically recruited for faces, Gauthier et al. (1999) invented a group of objects called greebles, which have three-dimensional structure and small features like faces. Before the participants in the study became familiar with the greebles, human faces produced a stronger fMRI response in the FFA than did the greebles. After weeks of 


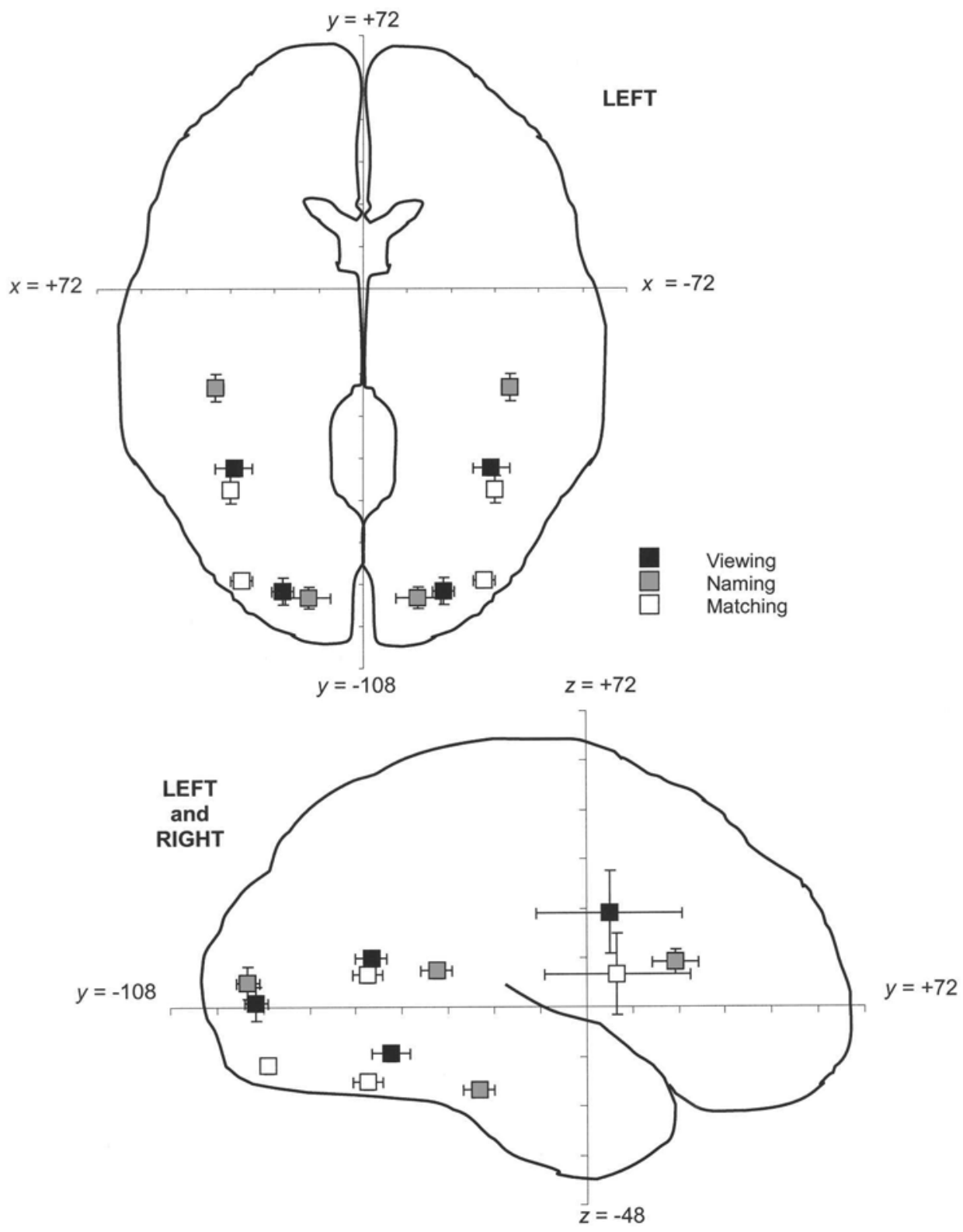

Figure 4. Mean activation loci for viewing (black squares), naming (gray squares), and matching (white squares) tasks overlaid on axial and sagittal slice representations. Standard error bars are shown.

training, the participants reached an "expert" level in identifying individual greebles. When the greeble experts were then imaged with $\mathrm{fMRI}$, the signal in response to sequential same/different matching of upright (vs. inverted) greebles rivaled the fMRI response to upright (vs. inverted) matching of faces in the mid-fusiform gyrus. The authors argued that this region is not necessarily dedicated to human face recognition but is instrumental in making fine distinctions among individuals of the same class of objects. Such a process requires identification at a subordinate or an individual level but is not exclusive for faces. One limitation of this study is that the analysis of fMRI responses was restricted to very specific ROIs that responded more strongly to faces than to objects. Results were not reported on the regions that were differentially activated by faces and greebles either before or after training. Nevertheless, this work highlights the idea that identification at an individuallevel of categorization may underlie face and object recognition dissociations (Gauthier et al., 1999).

Another factor that should be considered when interpreting brain activation associated with face recognition is the information processing engaged during recognition. The meta-analysis revealed that task effects explained 
Table 8

Percentages of Studies Reporting Activation in Different Brain Regions for the Three Categories

\begin{tabular}{lccc}
\hline & \multicolumn{3}{c}{ Object Category } \\
\cline { 2 - 4 } Brain Region & Natural & Manufactured & Faces \\
\hline Medial occipital & 25 & 30 & 25 \\
Posterior fusiform/ & & & \\
$\quad$ inferior occipital & 50 & 10 & 13 \\
Mid-fusiform & 38 & 30 & 88 \\
Anterior fusiform & 25 & 10 & 13 \\
Inferior temporal & 13 & 30 & 13 \\
Temporal pole & 29 & 11 & 20 \\
Superior temporal & 25 & 10 & 38 \\
Middle temporal & 0 & 20 & 13 \\
Inferior frontal & 50 & 50 & 50 \\
Premotor/precentral & 17 & 38 & 0 \\
Middle frontal & 17 & 13 & 50 \\
\hline
\end{tabular}

as much variance in brain activation patterns as did category effects. For example, naming tasks activate more anterior aspects of the ventral processing stream than do matching or viewing tasks. In addition, when studies of face recognition are examined on a case-by-case basis, it is interesting that the mid-fusiform region was implicated in all studies that employed face-matching and viewing tasks but was not necessarily implicated in facenaming tasks. Moreover, face-naming and face familiarity judgments tend to additionally activate the temporal pole (H. Damasio et al., 1996; Nakamura et al., 2000; Sergent et al., 1992). In fact, Nakamura et al. (2000) argued that the mid-fusiform region that responds strongly to faces is more likely to be involved in face perception but that the temporal pole region plays an important role in making familiarity judgments. Others have suggested that anterior temporal activation may be related to semantic retrieval (Moore \& Price, 1999; Tempini et al., 1998). Therefore, areas of the cortex that have been implicated in face recognition (e.g., the FFA and the temporal pole) may instead subserve perceptual or semantic processing, with faces making greater demands on these types of processing during visual recognition. In sum, when considering the cognitive function attributed to a particular brain region, one must also consider the cognitive demands of the recognition tasks used, rather than just the taxonomic category distinctions.

\section{Areas Specific to Natural Object Recognition}

Those fMRI and PET studies addressing the neural substrates for natural object recognition have contrasted pictures of animals versus tools (Chao et al., 1999; H. Damasio et al., 1996; Martin et al., 1996; Perani et al., 1999), animals versus unfamiliar faces (H. Damasio et al., 1996) animals, fruits, vegetables, or body parts versus vehicles, appliances, or tools and utensils (Moore \& Price, 1999; Smith et al., 2001), and names of animals versus names of tools (Cappa et al., 1998). The recognition tasks have included viewing, matching, silent naming, overt naming, and retrieving semantic information about an object referred to by a word.
Figure 1 graphically summarizes the Talairach coordinates in Tables 2 and 3 that are associated with natural object recognition (black boxes) in the occipital and ventral temporal cortices. Natural object recognition recruits more widely distributed areas in the occipital and ventral temporal cortices than does face or manufactured object recognition. Whereas activation associated with face recognition is largely concentrated in mid-fusiform regions, activation associated with natural object recognition is distributed across medial occipital, posterior fusiform, anterior fusiform, and temporal pole regions, with a higher concentration than other categories in medial occipital regions. Similar to face recognition, the activation for natural object recognition appears to be bilateral. Figure 2 summarizes the Talairach coordinates in Tables 4 and 5 that are associated with natural object activation in frontal and temporal regions (black boxes). Natural objects activate the superior temporal sulcus bilaterally and the right middle temporal gyrus. The frontal regions implicated in natural object recognition include left inferior frontal and right middle frontal, but the majority of activation foci are in occipital, ventral temporal, and more dorsal temporal regions.

Consistency of results across studies. Given the wider spatial distribution across cortical regions for activation foci associated with natural object recognition, less convergence occurs across studies in any particular cortical region, which contrasts sharply with the strong convergent findings in the mid-fusiform gyrus for faceprocessing tasks. From Table 8, the most convergence is in posterior fusiform and inferior frontal regions, but only half of the studies that examined natural object recognition versus other object classes reported activation in these regions. One region that has been more strongly implicated in natural object recognition, as compared with other categories, is the medial occipital cortex, as assessed with matching, naming, and viewing tasks (Chao et al., 1999; Martin et al., 1996). Indeed, Figure 1 supports this in showing a fairly strong concentration of natural-object-related activation in this region, but this site is only reported in two of the eight studies that could have detected activation in this region. Given the wide distribution of PET and fMRI responses to natural object recognition and the lack of convergence across studies in any particular brain region, there is little evidence that particular neural substrates are selectively involved in natural object recognition.

Analyses used to test category-specific hypotheses. The same concerns that were raised about hypothesis testing for face selectivity apply to natural object selectivity as well. Recall that the first step of the ROI approach is to create ROIs from the contrast of task versus baseline, collapsing across all categories of objects or across all tasks manipulated in the study. In the next step in the analysis, the relative contributions of the different categories to the strength of the signal within the ROIs are examined. For example, Chao et al. (1999) examined tool, house, animal, and face recognition tasks (viewing, 
matching, and naming) within the same study. The first step in hypothesis testing was to select ROIs that showed an increase in signal in response to all of the object categories combined versus the baseline task, which involved viewing phase-scrambled images of the objects. The next step was to isolate contiguous voxels (seven or more) within the ROIs isolated in the first step that showed a greater response to animals versus tools or to faces versus houses. The advantage of this type of ROI analysis is to ensure that the task of interest (natural object recognition in the present examples) produces a greater response than do both the baseline task (typically, visual fixation) and the control task (e.g., manufactured object recognition). Without the initial step of defining ROIs involved in the overall task relative to baseline, ROIs may be identified in which the baseline task produces a greater response than does the task of interest. Such an outcome would present difficulties in interpreting a region as being involved in object recognition. Although the ROI strategy ensures that the cortical regions that are included in the final interpretation are indeed task related, it does this at the expense of not being able to isolate regions that are selective for a given category. In other words, category differences isolated in this type of analysis are likely to be differences in magnitude of the signal, and not differences that demonstrate selectivity for a given category. Investigators using this strategy have clearly acknowledged that this ROI approach only allows conclusions about magnitude differences and does not allow conclusions about category-related cortical specialization (Chao et al., 1999; Haxby et al., 1999; Martin et al., 1996). Nevertheless, the distinction is reiterated here because it reflects the debate about whether semantic knowledge is organized into discrete cortical regions or whether the representations are more distributed across cortex.

Isolation of critical cognitive factors. The sensoryfunctional approach to category specificity posits that natural and manufactured object recognition relies on qualitatively different types of information: Natural object recognition is biased toward processing of visual features, such as shape, color, texture, and manufactured object recognition is biased toward processing of function, such as using a pencil for writing and using a fork for eating. Consequently, natural object recognition should make greater demands on regions involved in visual processing. Functional neuroimaging results from one group of investigators have been interpreted within this framework. The medial occipital activation that occurred with animal versus tool viewing, matching, and naming (Chao et al., 1999; Martin et al., 1996) was interpreted as top-down reactivation of the visual cortex to perform the additional visual analysis needed to differentiate animals from each other during naming. Although greater perceptual differentiation may be needed for natural object recognition than for manufactured object recognition, Martin et al. (1996) and Chao et al. did not directly manipulate the process of perceptual differentiation to sup- port that claim. A different group of investigators did not replicate the finding that natural objects make greater demands on the medial occipital cortex than do manufactured objects (Moore \& Price, 1999). Instead, Moore and Price showed greater left medial occipital activation in response to manufactured objects, relative to natural objects. In addition to manipulating object category (natural vs. manufactured), Moore and Price also manipulated structural complexity of the objects, where greater complexity was defined as a greater number of structural components. The complex objects included animals and complex manufactured objects, such as an airplane (which can be decomposed into several distinct parts), whereas the simple objects included fruits and simple manufactured objects, such as a hammer (which can typically be decomposed into only a few components). The contrast of complex versus simple objects for naming and word-picture matching tasks, regardless of category, produced activation in the right medial occipital cortex as well as in the right occipito-temporal-parietal junction (BA 19/39) and the right fusiform cortex (BA 20). Simple versus complex object naming and word-picture matching recruited the postcentral gyrus bilaterally. The greater activation of the right medial occipital cortex in response to complex objects may reflect additional visual processing, in line with Martin et al.'s (1996) proposal. Importantly, the nature of the additional visual processing was driven by complexity, not by category.

Gerlach et al. (1999) also examined the effects of greater perceptual differentiation on category-specific activation, using an object decision task, which required deciding whether a presented stimulus was a real object or not. In the "easy" discrimination condition, real natural and manufactured objects were presented with nonobjects, and in the "difficult" condition, real natural and manufactured objects were presented with chimeric nonobjects. Chimeric objects are composed of the parts of different objects, but as a whole, they do not form real objects themselves. Because chimeric and real objects share many of the same parts, differentiating the two types of stimuli is more difficult. Their analyses did not isolate different brain regions for natural and manufactured objects. However, the more difficult object decision task (real vs. chimeric objects) made greater demands on the right occipitotemporal cortex than did the easier object decision task (real objects vs. nonobjects). This difficulty-modulated occipito-temporal activation extended into anterior fusiform regions for natural object decision, but not for manufactured object decision. The authors argued that this right occipito-temporal activation reflects retrieval of stored visual knowledge needed to differentiate objects from nonobjects. Moore and Price (1999) reached a similar conclusion concerning the right occipito-temporal cortex. Although animals strongly recruited this region, this area also responded to greater visual complexity, suggesting that it is not specific to natural object recognition; rather, it is sensitive to increasing demands of structural processing. Notably, in both of these studies, taxo- 
nomic category differences were not necessarily stronger predictors of brain activation patterns than were the different demands on structural object processing.

In contrast to the right occipito-temporal cortex, left anterior temporal cortex activation may be specific to natural object recognition, given that it was more strongly activated when naming and matching noncolored drawings of natural objects, as compared with naming and matching tools and nonobjects (Moore \& Price, 1999). H. Damasio et al. (1996) also showed left, anterior temporal activation in response to natural object naming when contrasted with a face inversion decision task, but not when contrasted with tool naming. Other studies have not reported activation in this region, possibly because these brain regions were not imaged or were not analyzed.

In summary, several candidate regions have emerged as potential sites that might be strongly involved in natural object recognition, including the medial occipital, right occipito-temporal, and left anterior temporal cortices. In interpreting activation in these regions, investigators have suggested that greater demands on visual or structural object processing, rather than category membership, may explain the activation patterns. Likewise, the meta-analysis presented here indicated that in both occipital and ventral temporal regions, the recognition task used was also a strong predictor of patterns of brain activation. Taken together, these conclusions emphasize the need to examine the cognitive processing demands of the various recognition tasks in conjunction with a consideration of the object's category. Merely manipulating category membership as a variable in functional neuroimaging study designs does not necessarily allow one to conclude that a given type of processing is engaged during recognition. The approaches that have explicitly manipulated aspects of processing an object's structural descriptions are more promising in this regard.

\section{Areas Specific to Manufactured Object Recognition}

Neuroimaging studies investigating brain regions involved specifically in manufactured object recognition have compared tool with animal recognition (Cappa et al., 1998; Chao et al., 1999; H. Damasio et al., 1996; Martin et al., 1996; Perani et al., 1999), tool with unfamiliar face recognition (H. Damasio et al., 1996), tool recognition versus other object categories combined (Chao \& Martin, 2000), tool and vehicle recognition versus animal and fruit recognition (Moore \& Price, 1999), chair recognition versus other categories (Ishai et al., 2000), and house recognition versus other categories (Haxby et al., 1999; Ishai et al., 2000). The tasks used have included viewing, simultaneous matching, delayed match to sample, naming, and retrieval of semantic information from words.

The gray squares in Figures 1 and 2 depict the activation foci associated with manufactured object recognition in the occipital/ventral temporal cortex and in dorsal temporal/frontal regions, respectively. In the ventral temporal cortex, there is a preponderance of activation in the mid-fusiform cortex, with less activation in occipital or anterior temporal regions. The bulk of the mid-fusiform activation is situated somewhat more medially than the activation associated with other object categories. In frontal regions, there is a clear focus for manufactured object recognition in left inferior frontal/precentral regions. Also, manufactured objects appear to activate the left posterior middle temporal cortex more so than do other object categories.

Consistency of results across studies. Three studies have consistently reported mid-fusiform activation in response to different categories of manufactured objects (Chao et al., 1999; Haxby et al., 1999; Ishai et al., 2000). Moreover, this activation is more medial than the activation associated with either natural objects or faces (see Figures 1 and 3). However, more lateral activation-in the inferior temporal gyrus - has been reported in response to tools (H. Damasio et al., 1996) or chairs (Ishai et al., 2000). Nevertheless, the meta-analysis conducted only for ventral temporal regions confirmed the trend for more medial mid-fusiform activation in response to manufactured objects, as compared with recognition of other object categories. Four studies have reported manufacturedobject-related activation in the left inferior frontal and left precentral gyrus (also referred to as the premotor cortex), as is shown in Figure 2. None of those studies reported activation in homologous right-hemisphere structures in response to manufactured objects. In addition, the left precentral activation is almost exclusively related to tool recognition, whereas the left inferior frontal activation is also associated with face and natural object recognition. In posterior middle temporal regions, two studies have reported activation in the left hemisphere (Chao et al., 1999; Martin et al., 1996), and one study has reported activation in the homologous right-hemisphere region (Chao et al., 1999). All in all, the studies have converged in finding activation in the medial fusiform gyrus, the left precentral gyrus, and the left posterior middle temporal cortex in response to manufactured object recognition, but the meta-analysis showed that manufactured objects dissociate from other object categories only in ventral, middle, and superior temporal regions.

Analyses used to test category-specific hypotheses. Again, the consistent findings for manufactured object recognition must be considered with respect to the analyses used and the regions of the brain that were imaged. The three studies that showed consistent medial fusiform activation in response to manufactured objects first isolated an area in the fusiform cortex that responded more strongly to all of the categories combined, as compared with a baseline task (Chao et al., 1999; Haxby et al., 1999; Ishai et al., 2000). However, manufactured objects (houses, chairs, and tools) tended to activate the medial extent of this preselected region more strongly than did the other categories manipulated (faces and animals). This demonstrates differences in magnitude of brain activation for different object categories but does not demonstrate selectivity for manufactured objects. Of the four studies re- 
porting activation in the left precentral gyrus (or the left premotor cortex) in response to tools, two of those first isolated regions that were responsive to the recognition task for all categories of objects used in the study (Chao \& Martin, 2000; Perani et al., 1999). With regard to the activation associated with manufactured object recognition in the posterior temporal cortex, one of the two studies (Chao et al., 1999) selected this region on the basis of the fact that it was activated by all categories of objects, relative to baseline, in naming and viewing tasks. In sum, then, many of the studies have demonstrated stronger responses to manufactured objects than to other categories in particular brain regions, but the analyses used have not permitted strong conclusions that a single area or areas are selectively recruited for manufactured object recognition.

Isolation of critical cognitive factors. A common interpretation of the activation in left premotor regions in response to manufactured objects is that they are involved in storing information about, or motor programs related to, actions performed on manipulable manufactured objects. In fact, all of the left frontal activation is reported in contrasts that compared recognition of tools with that of other objects (Chao \& Martin, 2000; Grabowski et al., 1998; Martin et al., 1996; Perani et al., 1999), rather than in contrasts comparing other manufactured categories with other objects. For example, Chao and Martin (2000) imaged frontal regions and showed that the response in the left premotor cortex was indeed stronger for tools than for other manufactured objects (houses) or other natural objects (faces and animals), as assessed with viewing and naming tasks. In that study, tools also more strongly activated posterior parietal regions, as compared with other categories (including houses). These investigators argued that ventral premotor and posterior parietal regions store information about how to act on graspable objects, such as tools. Therefore, these regions are crucial for recognizing this category of manufactured objects.

Although this interpretation is reasonable, there was no direct demonstration in Chao and Martin's (2000) study that manipulability is the factor driving activation in the premotor cortex. However, at least three other studies have linked the left premotor cortex with the retrieval of information about tool use. Grafton, Fadiga, Arbib, and Rizzolatti (1997) showed that the left premotor cortex (both dorsal and ventral aspects) was activated when participants silently named the uses for tools, across several different baseline conditions. In addition, the ventral premotor region was more selective for tool use naming than was the dorsal premotor region, which responded when tools were simply viewed or silently named. Martin, Haxby, Lalonde, Wiggs, and Ungerleider (1995) showed that when actions for objects were retrieved from memory, the left inferior frontal gyrus near Broca's area was activated. Although this activation was not centered in the premotor cortex, it was more extensive when actions were retrieved from pictures than from words, and the posterior extent of this picture-induced activation may have included the ventral premotor cortex. In another study (Moll et al., 2000), individuals heard the name of an object and pantomimed with their hands the action that would be performed on that object. In the control condition, the participants simply executed a series of hand movements that were not pantomimes. In addition to actually executing the motor programs for the pantomimes, the participants also imagined the actions that would be performed on the objects without any hand movements. Both real and imagined tool use movements activated similar regions of the cortex-namely, the intraparietal sulcus in the left posterior parietal cortex and the left posterior dorsolateral frontal cortex. These results are similar to those of Chao and Martin (2000), except that the activation for real and imagined movements in the left premotor cortex may have been more dorsal than was the activation associated with the recognition of tools (however, no Talairach coordinates were provided by Moll et al., so this issue cannot be fully addressed).

In summary, the premotor activation in response to tools may be involved in retrieving stored motor programs for the actions associated with tools. However, the individual studies conducted on this topic thus far have manipulated only a subset of the variables that may be driving left premotor activation. For example, only one study (Chao \& Martin, 2000) has compared manipulable with nonmanipulable manufactured objects, but this study used a naming task, rather than a task that required retrieval of information about actions associated with tools. The other studies that did focus on retrieval or execution of actions associated with tools (Grafton et al., 1997; Martin et al., 1995; Moll et al., 2000) did not also manipulate object category. A study that dissociates manufactured from natural object or face recognition along the dimension of imagining or retrieving actions associated with objects would be a stronger test of the proposal that the left premotor cortex is activated during tool recognition because it stores information about actions associated with tools.

A similar interpretation has been provided for the consistent activation in the left posterior middle temporal cortex. Given the proximity of this region to visual motion processing area V5/MT, Martin et al. (1995) suggested that it is activated during retrieval of actions associated with objects because these actions or uses have characteristic motion patterns. In fact, a more recent interpretation of this finding extends the idea to motion associated with natural objects as well. Martin, Ungerleider, and Haxby (2000) have proposed an organization in the middle temporal cortex in which more dorsal aspects respond to nonbiological motion and more ventral aspects respond to biological motion. This proposal, however, is not based on a direct demonstration of dissociated brain activation patterns for biological and nonbiological motion in the same regions that are activated by natural and manufactured objects, respectively.

The more medial mid-fusiform activation that emerges for the recognition of manufactured objects, as compared with other, natural object categories, which was a prominent effect in the present analysis, has been interpreted 
with respect to an organization based on form differences among different categories of objects (Martin et al., 2000). As was discussed earlier, differences in the processing of object structure may explain patterns of brain activation in the ventral temporal cortex (Gerlach et al., 1999; Moore \& Price, 1999). However, the particular aspect of form processing that is relevant to the more medial midfusiform activation in response to manufactured objects has yet to be revealed. One possibility is that manufactured objects, especially tools, may be decomposed into fewer components than are animals or faces and are, consequently, simpler in structure. But Moore and Price directly manipulated the factor of structural complexity and showed that simpler objects selectively activated the postcentral gyrus bilaterally. No differences were observed in mid-fusiform regions. Another aspect of object structure that could differentiate between manufactured objects and other categories is that manufactured objects share the same structural components in common but are differentiated by the spatial arrangement of those components. Natural objects, in contrast, overlap in terms of both the components and their spatial arrangement but are differentiated by the relative sizes of the components (Sacchett \& Humphreys, 1992). In a similar vein, Farah (1994) has suggested that some categories, such as objects, can be more readily decomposed into parts, whereas other categories, such as faces, resist decomposition. Although these proposals have been put forth to explain category differences in the context of category-specific visual agnosia, they have not yet been tested with functional neuroimaging techniques.

\section{Summary and Conclusions}

In the present review, functional neuroimaging studies of category-specific processing were critically examined, and a meta-analysis of stereotactic coordinates was conducted. Tables 2-5 reveal some overall patterns of dissociated brain activation for different object categories, but the meta-analyses confirmed only some of these patterns. Object category predicted patterns of PET or fMRI responses in the ventral temporal cortex and the superior and middle temporal cortex. In general, manufactured object recognition dissociates from natural object and face recognition in temporal regions, but not in frontal or occipital regions. Some dissociations of face recognition from manufactured object recognition were also observed in the temporal cortex, but natural object recognition rarely dissociated from recognition of other object categories.

How well do category-related patterns of brain activation map on to lesion patterns that underlie category-specific impairments? This comparison is difficult, because the lesion patterns associated with category-specific impairments are not always clear (Caramazza \& Shelton, 1998; McCarthy \& Warrington, 1990) and the lesion sites are often localized only to a particular lobe (e.g., left temporal region, Sheridan \& Humphreys, 1993). When category differences emerged in the present analysis, the spatial separation of the localization was less than $1 \mathrm{~cm}$, which is a much finer spatial resolution than can be de- scribed in lesion studies. In perhaps the largest group study of lesion sites that underlie category-specific naming impairments, H. Damasio et al. (1996) showed that face-naming deficits were associated with left temporal pole lesions, natural-object-naming deficits were associated with lesions to the left, anterior aspects of the inferior temporal cortex, and manufactured-object-naming deficits were associated with lesions to the left, posterior temporal cortex at the junction of the occipital and the parietal lobes. Somewhat confirming a more anterior locus for face naming in the present analysis, functional activation associated with face recognition tasks was, on average, more anterior than activation associated with natural or manufactured objects in ventral temporal regions (Figure 3); however, this difference did not reach statistical significance. One limitation of many of the functional neuroimaging studies reviewed here is that imaging protocols were restricted to posterior brain regions, or analyses were restricted to particular ROIs. This would obviously not reveal the entire spatial extent of categoryrelated brain activation patterns.

In addition to brain activation patterns' being driven by category membership in the present analysis, an equally prominent effect was the type of recognition task used. The meta-analysis revealed that matching tasks tend to activate more inferior occipital sites than do either naming or viewing tasks. Another task difference that emerged from the meta-analysis was that naming tasks recruit more anterior fusiform sites than do either matching or viewing tasks. This shift in activation locus for naming may reflect the greater semantic demands of naming, as compared with either viewing or matching.

To supplement the meta-analysis of stereotactic coordinates, the present paper critically reviewed functional neuroimaging studies of category specificity along three dimensions. First, how consistent are the findings across studies for category-specific cortical localization? The strongest convergent finding was the activation of the mid-fusiform gyrus in response to faces, which was reported by $88 \%$ of the studies that imaged this region. However, no more than $50 \%$ convergence was found for either natural or manufactured object recognition in any brain region. Consequently, to date, there is little convergence across studies for category-specific brain activation patterns. This conclusion should be tempered by the fact that the number of studies sampling particular brain regions-particularly, the frontal cortex-is quite small.

The second question used to evaluate category-specific patterns of brain activation was whether the statistical analyses have allowed for testing the hypothesis that certain cortical regions are selectively involved in recognizing a particular object category. In general, many of the regions isolated in these studies are selected on the basis that they respond to all object categories manipulated in the particular study, relative to a baseline or control task. Subsequent analyses then examine the contribution of the separate object categories to the $\mathrm{fMRI}$ response in these predefined areas. This is a reasonable approach, but it does not allow conclusions about category-related selec- 
tivity-it merely allows conclusions concerning differences in magnitude of PET and fMRI responses. Importantly, not all theoretical frameworks would predict that discrete cortical regions are selectively involved in recognizing different object categories. Instead, object representations may be more widely distribu ${ }^{2}$ ed acros- the cortex, and the organizing principle may reflect differences in the processing of object attributes, rather than category membership itself (Mummery et al., 1998). Therefore, demonstrating differences in the magnitude of brain activation for different object categories, but not differences in response selectivity, should not be considered a shortcoming. The distinction is emphasized here because of the theoretical implications of discrete versus distributed and overlapping object representations in the cortex. Unfortunately, the analyses used in some functional neuroimaging approaches do not sufficiently test this hypothesis.

The final question asked whether functional neuroimaging studies have addressed the important factors that are posited to underlie category-specific object processing, as outlined by cognitive neuropsychological approaches. The answer is that, to date, most studies have focused on manipulations of category membership without focusing on the type of processing engaged when different classes of objects are recognized. Some recent exceptions that have tested the claim that natural object recognition requires more visual or structural processing than does manufactured object recognition have shown that the processing of object structure is as predictive, if not more predictive, of brain activation patterns as is category membership (Gerlach et al., 1999; Moore \& Price, 1999). Studies such as these, which devise tasks that make differential demands on perceptual or structural processing, may be more promising than studies that simply manipulate object category without regard to the processing demands of the task. Humphreys and Riddoch (1987) have made this point in the context of interpreting human lesion studies of category specificity—namely, characterizing the cognitive processing engaged while different object categories are recognized may be a more fruitful approach to understanding category dissociations. The meta-analysis in the present review article reemphasizes this point by showing that the type of recognition task was strongly predictive of brain activation patterns. Consequently, functional neuroimaging approaches to category-specific cortical organization may need to shift the focus from designs that emphasize strict delineations of category membership to designs that focus on the degree and type of information processing involved during visual recognition.

\section{REFERENCES}

Allison, T., Ginter, H., McCarthy, G., Nobre, A., Puce, A., Luby, M., \& SPENCER, D. D. (1994). Face recognition in human extrastriate cortex. Journal of Neurophysiology, 71, 821-825.

Bauer, R. M. (1993). Agnosia. In K. M. Heilman \& E. Valenstein (Eds.), Clinical neuropsychology (3rd ed., pp. 215-273). New York: Oxford University Press.
Bruce, V., \& Humphreys, G. W. (1994). Recognizing objects and faces. Visual Cognition, 1, 141-180.

Cappa, S. F., Perani, D., Schnur, T., Tettamanti, M., \& Fazio, F. (1998). The effects of semantic category and knowledge type on lexicalsemantic access: A PET study. NeuroImage, 8, 350-359.

Caramazza, A., \& Shelton, J. R. (1998). Domain-specific knowledge systems in the brain: The animate-inanimate distinction. Journal of Cognitive Neuroscience, 10, 1-34.

Chao, L. L., Haxby, J. V., \& Martin, A. (1999). Attribute-based neural substrates in temporal cortex for perceiving and knowing about objects. Nature Neuroscience, 2, 913-919.

Chao, L. L., \& Martin, A (2000). Representation of manipulable manmade objects in the dorsal stream. NeuroImage, 12, 478-484.

Damasio, A. R., Damasio, H. \& Van Hoesen, G. W. (1982). Prosopagnosia: Anatomic basis and behavioral mechanisms. Neurology, 32, 331-341.

Damasio, A. R. Tranel, D., \& Damasio, H. D. (1990). Face agnosia and the neural substrates of memory. Annual Review of Neuroscience, 13, 89-109.

Damasio, H., Grabowski, T. J., Tranel, D., Hichwa, R. D., \& Damasio, A. R. (1996). A neural basis for lexical retrieval. Nature, 380, 499-505.

FARAH, M. J. (1990). Visual agnosia: Disorders of object recognition and what they tell us about normal vision (4th ed.). Cambridge, MA: MIT Press.

FARAH, M. J. (1994). Specialization within visual object recognition: Clues from prosopagnosia and alexia. In M. J. Farah \& G. Ratcliff (Eds.), The neuropsychologyof high-level vision (pp. 133-146). Hillsdale, NJ: Erlbaum.

FARAH, M. J., \& Aguirre, G. K. (1999). Imaging visual recognition: PET and fMRI studies of the functional anatomy of human visual recognition. Trends in Cognitive Sciences, 3, 179-186.

Forde, E., \& Humphreys, G. W. (1999). Category-specific recognition impairments: A review of important case studies and influential theories. Aphasiology, 13, 169-193.

Gaffan, D., \& HeYwood, C. A. (1993). A spurious category-specific visual agnosia for living things in normal human and non-human primates. Journal of Cognitive Neuroscience, 5, 118-128.

Gauthier, I., Skudlarski, P., Gore, J. C., \& Andersen, A. W. (2000). Expertise for cars and birds recruits brain areas involved in face recognition. Nature Neuroscience, 3, 191-197.

Gauthier, I., Tarr, M. J., Anderson, A. W., Skudlarski, P., \& Gore, J. C. (1999). Activation of the middle fusiform 'face area' increases with expertise in recognizing novel objects. Nature Neuroscience, $\mathbf{2}$, $568-573$.

Gauthier, I., Tarr, M., J., Moylan, J., Skudlarski, P., Gore, J. C., \& ANDERson, A. W. (2000). The fusiform "face area" is part of a network that processes faces at the individual level. Journal of Cognitive Neuroscience, 12, 495-504.

Gerlach, C., Law, I., Gade, A., \& Paulson, O. B. (1999). Perceptual differentiation and category effects in normal object recognition. Brain, 122, 2159-2170.

Grabowski, T. J., Damasio, H., \& Damasio, A. R. (1998). Premotor and prefrontal correlates of category-related lexical retrieval. NeuroImage, 7, 232-243.

Grafton, S. T., Fadiga, L., Arbib, M. A., \& Rizzolatti, G. (1997). Premotor cortex activation during observation and naming of familiar tools. NeuroImage, 6, 231-236.

Haxby, J. V., Horwitz, B., Ungerleider, L. G., Maisog, J. M., PieTRINI, P., \& GRADY, C. L. (1994). The functional organization of human extrastriate cortex: A PET-rCBF study of selective attention to faces and locations. Journal of Neuroscience, 14, 6336-6353.

Haxby, J. V., Ungerleider,L. G., Clark, V. P., Schouten, J. L., Hoffman, E. A., \& MARTIN, A. (1999). The effect of face inversion on activity in human neural systems for face and object perception. Neuron, 22, 189-199.

Humphreys, G. W., \& RidDoch, M. J. (1987). On telling your fruit from your vegetables: A consideration of category-specific deficits after brain damage. Trends in Neurosciences, 10, 145-148.

Humphreys, G. W., Riddoch, M. J., \& Quinlan, P. T. (1988). Cascade processes in picture identification. Cognitive Neuropsychology, 5, 67-103. 
Ishai, A., Ungerleider, L. G., Martin, A., \& Haxby, J. V. (2000). The representation of objects in the human occipital and temporal cortex. Journal of Cognitive Neuroscience, 12 (Suppl. 2), 35-51.

Jolicteur, P., Gluck, M. A., \& Kosslyn, S. M. (1984). Pictures and names: Making the connection. Cognitive Psychology, 16, 243-275.

Kanwisher, N., McDermott, J., \& Chun, M. M. (1997). The fusiform face area: A module in human extrastriate cortex specialized for face perception. Journal of Neuroscience, 17, 4301-4311.

Krieman, G., Koch, C., \& Fried, I. (2000). Category-specific visual responses of single neurons in the human medial temporal lobe. Nature Neuroscience, 3, 946-953.

Kurbat, M. A., \& FArah, M. J. (1998). Is the category-specific deficit for living things spurious? Journal of Cognitive Neuroscience, 10, 355-361.

Martin, A., Haxby, J. V., Lalonde, F. M., Wiggs, C. L., \& UngerLEIDER, L. G. (1995). Discrete cortical regions associated with knowledge of color and knowledge of action. Science, 270, 102-105.

Martin, A., Ungerleider, L. G., \& Haxby, J. V. (2000). Category specificity and the brain: The sensory/motor model of semantic representations of objects. In M. S. Gazzaniga (Ed.), The new cognitive neurosciences (2nd ed., pp. 1023-1036). Cambridge, MA: MIT Press.

Martin, A., Wiggs, C. L., Ungerleider, L., \& Haxby, J. (1996). Neural correlates of category-specific knowledge. Nature, 379, 649-652.

McCARthy, R. A., \& Warrington, E. K. (1990). Cognitive neuropsychology: A clinical introduction. New York: Academic Press.

Moll, J., de Oliveira-Souza, R, Passman, L. J., Cunha, F. C., SouzaLima, F., \& ANDREIUOLO, P. A. (2000). Functional MRI correlates of real and imagined tool-use pantomimes. Neurology, 54, 1331-1336.

Moore, C. J., \& Price, C. J. (1999). A functional neuroimaging study of the variables that generate category-specific object processing differences. Brain, 122, 943-962.

Mummery, C. J., Patterson, K., Hodges, J. R., \& Price, C. J. (1998). Functional neuroanatomy of the semantic system: Divisible by what? Journal of Cognitive Neuroscience, 10, 766-777.

Nakamura, K., Kawashima, R, Sato, N., Nakamura, A, Sugiura, M., Kato, T., Hatano, K., Ito, K., Fukuda, H., Schormann, T., \& Zilles, K. (2000). Functional delineation of the human occipitotemporal areas related to face and scene processing: A PET study. Brain, 123, 1903-1912.

Pallis, C. A. (1955). Impaired identification of faces and places with agnosia for colours. Journal of Neurology, Neurosurgery \& Psychiatry, 18, 218-224.

Perani, D., Schnur, T., Tettamanti, M., Gorno-Tempini, M., Cappa, S. F., \& FAZIO, F. (1999). Word and picture matching: A PET study of semantic category effects. Neuropsychologia, 37, 293-306.

Puce, A., Allison, T., Asgari, M., Gore, J. C., \& McCarthy, G. (1996). Differential sensitivity of human visual cortex to faces, letterstrings, and textures: A functional magnetic resonance imaging study. Journal of Neuroscience, 16, 5205-5215.

Puce, A., Allison, T., Gore, J. C., \& McCarthy, G. (1995). Facesensitive regions in human extrastriate cortex studies by functional MRI. Journal of Neurophysiology, 74, 1192-1199.

Rosch, E., Mervis, C. B., Gray, W. D., Johnson, D. M., \& BoyesBraem, P. (1976). Basic objects in natural categories. Cognitive Psychology, 8, 382-439.

Rossion, B., Gauthier, I., Tarr, M. J., Bruyer, R. Linotte, S., \& Crommelinck, M. (2000). The N170 occipito-temporal component is delayed and enhanced to inverted faces but not to inverted objects: An electrophysiological account of face-specific processes in the human brain. NeuroReport, 11, 69-74.
Sacchett, C., \& Humphreys, G. W. (1992). Calling a squirrel a squirrel but a canoe a wigwam: A category-specific deficit for artefactual objects and body parts. Cognitive Neuropsychology, 9, 73-86.

Sergent, J., Ohta, S., \& MacDonald, B. (1992). Functional neuroanatomy of face and object processing: A positron emission tomography study. Brain, 1, 15-36.

SheridAN, J., \& Humphreys, G. W. (1993). A verbal-semantic categoryspecific recognition impairment. Cognitive Neuropsychology, 10, 143-184.

Smith, C. D., Anderson, A. H., Kryscio, R. J., Schmitt, F. A., Kindy, M. S., Blonder, L. X., \& Avison, M. J. (2001). Differences in functional magnetic resonance imaging activation by category in a visual confrontation naming task. Journal of Neuroimaging, 11, 165-170.

Spitzer, M., Kischia, U., Guckel, F., Bellemann, M. E., Kammer, T., Seyyedi, S., Weisbrod, M., Schwartz, A., \& Brix, G. (1998). Functional magnetic resonance imaging of category-specific cortical activation: Evidence for semantic maps. Cognitive Brain Research, $\mathbf{6}$, 309-319.

Spitzer, M., Kwong, K. K., Kennedy, W., Rosen, B. R., \& BelliVEAU, J. W. (1995). Category-specific brain activation in fMRI during picture naming. NeuroReport, 6, 2109-2112.

Talairach, J., \& Tournoux, P. (1988). Co-planar stereotaxic atlas of the human brain (M. Rayport, Trans.). New York: Thieme Medical Publishers.

Tempini, M. L. G., Price, C. J., Josephs, O., Vandenberghe, R., Cappa, S. F., Kapur, N., \& Frackowiak, R. S. J. (1998). The neural systems sustaining face and proper-name processing. Brain, 121, 2103-2118.

Thompson-Schill, S. L., Aguirre, G. K., D’Esposito, M., \& Farah, M. J. (1999). A neural basis for category and modality specificity of semantic knowledge. Neuropsychologia, 37, 671-676.

Tranel, D., \& Damasio, A. R. (1999). The neurobiology of knowledge retrieval. Behavioral \& Brain Sciences, 22, 303.

Tranel, D., Damasio, H., \& Damasio, A. R. (1997). A neural basis for the retrieval of conceptual knowledge. Neuropsychologia, 35, 13191327.

Tranel, D., Logan, C., Frank, R. J., \& Damasio, A. R. (1997). Explaining category-related effects in the retrieval of conceptual and lexical knowledge for concrete entities: Operationalization and analysis of factors. Neuropsychologia, 32, 1329-1339.

Warrington, E., \& McCARThy, R. A. (1994). Multiple meaning systems in the brain: A case for visual semantics. Neuropsychologia, 32, 1465-1473.

Warrington, E., \& Shallice, T. (1984). Category specific semantic impairments. Brain, 107, 829-854.

\section{NOTES}

1. The MANOVA treated all of the Talairach coordinates as independent observations in a between-factors design, rather than treating the coordinates as repeated measures from the same study. A repeated measures design would be nearly impossible to conduct, because not every study manipulated the same variables and imaged the same brain regions, which would leave many cells of a repeated measures design empty.

2. Tamahane's F2 test was chosen because it does not assume equal variances, which is important given that the present design was unbalanced.

(Manuscript received January 1, 2001; revision accepted for publication May 15, 2001.) 